\title{
Consistent baryon mapping of quark systems
}

\author{
S. Pittel \\ Bartol Research Institute, University of Delaware, Newark, Delaware 19716 \\ J. M. Arias \\ Departamento de Física Atómica, Molecular y Nuclear, Universidad de Sevilla, Apdo. 1065, 41080 Sevilla, Spain \\ J. Dukelsky \\ Grupo de Física Nuclear - Facultad de Ciencias, Universidad de Salamanca, 37008 Salamanca, Spain \\ A. Frank \\ Instituto de Ciencias Nucleares and Laboratorio de Cuernavaca, Instituto de Física, \\ Universidad Nacional Autónoma de México, Apdo. Postal 70-543, 04510 México, Distrito Federal, México
}

(Received 7 December 1993)

\begin{abstract}
We present a new and consistent mapping of colorless three-quark clusters onto colorless triplet fermions (baryons) and test it in the context of a three-color extension of the Lipkin model. For systems with two triplets (for which the problem can be solved without approximation both before and after the mapping), we exactly reproduce the dynamics of the model for the variety of correlation structures considered.
\end{abstract}

PACS number(s): 21.60.Gx, 21.60.Fw, 21.30.+y, 12.39.-x

\section{INTRODUCTION}

Establishing a connection between nuclear physics and QCD has been an area of intense research in the last few years. Central to this effort is the goal of isolating quark effects in nuclei.

In recent years, constituent quark models [1] have been applied with considerable success to nuclear systems with few particles. While many questions remain concerning the validity of such an approach (e.g., the lack of a connection to QCD and its apparent violation of the underlying physics of chiral symmetry and spontaneous chiral symmetry breaking processes), in view of these successes it seems worthwhile to develop these models further, in order to see whether they can provide a partial bridge between the physics of QCD and that of finite nuclei.

At present, such models have been directly applied to one- and two-baryon systems only. To treat systems with larger numbers of nucleons, it has proven necessary to introduce approximations. One possibility that has been explored is to use the resonating group method in the sixquark problem to extract an effective nucleon-nucleon interaction, which is subsequently diagonalized in the space of several nucleons [2]. Unfortunately, many-quark effects that may arise when more than two nucleons are present will be missed in such an approach. To incorporate them, we would like to bypass the two-nucleon problem and work directly in the space of many quarks. Since a system of $3 A$ quarks will cluster into $A$ triplets (nucleons) at normal nuclear densities, a necessary ingredient in any such approach is a method for handling strong three-body correlations in a many-body environment.

Recently, it has been suggested [3-5] that mapping methods [6] might provide a practical means of accomplishing this. The basic idea is to map colorless threequark clusters, which do not satisfy exact fermion anticommutation rules, onto triplet fermions (baryons) that do. Such a mapping leads from the original multiquark Hamiltonian to an effective Hamiltonian for these baryons, which rigorously incorporates the physics of the Pauli principle at the quark level.

The virtue of this approach is not in a reduction of the degrees of freedom - there are in fact more states in the mapped space than in the original space - but rather in the representation of the dynamics as a system of interacting baryons. These baryons contain as a subspace the physical nucleons (as well as excited nucleonic states) and interact with one another in ways that should be amenable to the usual fermion many-body techniques [7], e.g., Hartree-Fock, Tamm-Dancoff, and random phase approximations, Brueckner theory, etc.

Several different mappings of quarks to triplet fermions have been recently discussed. Pittel, Engel, Dukelsky, and Ring [3] (hereafter referred to as PEDR) proposed a two-step procedure, whereby pairs of quarks are first mapped onto diquark bosons and then boson-fermion pairs are mapped onto triplet fermions. Despite the success of this mapping in reproducing the dynamics of the test model to which it was applied, it nevertheless has some drawbacks. On the one hand, it does not lead directly to triplet fermions that are antisymmetric in their three indices. In addition, as formulated, it can only be applied to quark Hamiltonians dominated by two-body interactions. At roughly the same time, Nadjakov [4] suggested an alternative mapping that leads directly to antisymmetric triplet fermions and, furthermore, is applicable to three-body interactions. His mapping, how- 
ever, is not appropriate for systems that are dominated by two-body interactions. Clearly, what is needed is a mapping that consistently treats both two- and threequark interactions.

One such method was in fact proposed soon thereafter by Meyer [5]. However, this mapping does not seem to properly treat two-body interactions either, at least when a truncation to colorless triplet fermions is imposed. Building on the ideas of Meyer, we have now succeeded in formulating a new baryon mapping of (colorless) three-quark clusters that seems to satisfy all the desired requirements. In Sec. II, we briefly review some general features of baryon mappings and present our colorless version.

Both PEDR and Meyer tested their mappings in the context of an exactly solvable model of quarks often referred to as the Bonn quark shell model (BQSM) [8]. While this model has some attractive features, it also has some serious limitations. Perhaps the most significant is that it does not produce spatially localized colorless three-quark clusters (i.e., nucleons) [9]. Thus a second goal of this work has been to develop an alternative quark model on which to test our mapping. The model that we have chosen is a three-color extension of the well-known Lipkin model [10], which in its traditional version has been used extensively to test various nuclear many-body techniques. We describe this model in Sec. III and discuss its algebraic solution for small numbers of particles. A crucial component of this model is that it admits, for different values of its parameters, dynamical one-, two-, and three-body correlations.

In Sec. IV, we apply our mapping to the three-color Lipkin model for two triplets and present the results. The bottom line is that the mapping seems to work perfectly, when all colorless baryon states are included. For systems involving a larger number of triplets, this is clearly not possible. Thus, in Sec. V, where we summarize the principal conclusions of our work, we also describe some future extensions needed to further test the applicability of our methods for many-triplet systems.

\section{BARYON MAPPINGS OF QUARK SYSTEMS}

\section{A. Preliminaries}

Our starting point is a nonrelativistic model of constituent quarks. We denote the quark creation and annihilation operators of the model by $q_{c i}^{\dagger}$ and $q_{c i}$, respectively. The first subscript denotes the color quantum number and the second denotes all the rest. These operators satisfy the usual fermion anticommutation relation

$$
\left\{q_{c i}, q_{c^{\prime} i^{\prime}}^{\dagger}\right\}=\delta_{c i, c^{\prime} i^{\prime}} \equiv \delta_{c c^{\prime}} \delta_{i i^{\prime}} .
$$

QCD considerations suggest that the quark Hamiltonian may include up to three-body interactions, all of which are color scalars. Such a Hamiltonian can always be expressed in terms of the following colorless operators:

$$
\begin{gathered}
A_{a b}=\sum_{1} q_{1 a}^{\dagger} q_{1 b} \\
B_{a b c d}=\sum_{12345} \epsilon_{123} \epsilon_{145} q_{2 a}^{\dagger} q_{3 b}^{\dagger} q_{5 d} q_{4 c}
\end{gathered}
$$

and

$$
C_{a b c d e f}=\sum_{123456} \epsilon_{123} \epsilon_{456} q_{1 a}^{\dagger} q_{2 b}^{\dagger} q_{3 c}^{\dagger} q_{6 f} q_{5 e} q_{4 d} .
$$

Here, and in the subsequent analysis, we denote the color indices by numbers and the rest by roman letters.

The idea of a baryon mapping is to replace the system of interacting quarks by an equivalent one of interacting triplet fermions. We denote the creation and annihilation operators of the resulting (mapped) space by $\Lambda_{1 a 2 b 3 c}^{\dagger}$ and $\Lambda_{1 a 2 b 3 c}$, respectively. They satisfy the multi-index anticommutation relation

$$
\left\{\Lambda_{1 a 2 b 3 c}, \Lambda_{4 d 5 e 6 f}^{\dagger}\right\}=\delta(1 a 2 b 3 c, 4 d 5 e 6 f)
$$

where

$$
\begin{aligned}
\delta(1 a 2 b 3 c, 4 d 5 e 6 f)= & \delta_{1 a, 4 d} \delta_{2 b, 5 e} \delta_{3 c, 6 f}+\delta_{1 a, 5 e} \delta_{2 b, 6 f} \delta_{3 c, 4 d}+\delta_{1 a, 6 f} \delta_{2 b, 4 d} \delta_{3 c, 5 e} \\
& -\delta_{1 a, 4 d} \delta_{2 b, 6 f} \delta_{3 c, 5 e}-\delta_{1 a, 5 e} \delta_{2 b, 4 d} \delta_{3 c, 6 f}-\delta_{1 a, 6 f} \delta_{2 b, 5 e} \delta_{3 c, 4 d}
\end{aligned}
$$

The operator $\Lambda_{1 a 2 b 3 c}^{\dagger}$, by definition, creates a baryon corresponding to three quarks in the states $1 a, 2 b$, and $3 c$. This correspondence is expressed through the requirement that both $\Lambda_{1 a 2 b 3 c}^{\dagger}$ and $\Lambda_{1 a 2 b 3 c}$ are antisymmetric under interchanges of their quark indices, e.g., $\Lambda_{1 a 2 b 3 c}^{\dagger}=-\Lambda_{2 b 1 a 3 c}^{\dagger}$, etc.

The space generated by these baryon operators is in fact larger than that of the original quarks. This can be seen by considering the state of two baryons,

$$
|1 a 2 b 3 c, 4 d 5 e 6 f\rangle_{B}=\Lambda_{1 a 2 b 3 c}^{\dagger} \Lambda_{4 d 5 e 6 f}^{\dagger}|0\rangle_{B}
$$

where the subscript $B$ refers to states in the baryon space. The state (2.7) is antisymmetric under the interchange of the indices corresponding to any two quarks within one of the two triplets (e.g., $1 a$ with $2 b$ or $4 d$ with $5 e$ ) and also under the interchange of one triplet with the other. However, it is not antisymmetric under the interchange of the quantum numbers of a quark in one triplet (e.g., 1a) with those of a quark in the other (e.g., $4 d$ ). A fully antisymmetric two-triplet state may be recovered by taking an appropriate linear combination of the states (2.7). As a consequence, there is indeed a subset of two-triplet (and likewise many-triplet) states that are fully antisymmetric and, furthermore, are in one-to-one correspondence with the states of the original quark space. This is referred to as the physical subspace. There is, however, another class of states that are not fully antisymmetric under interchange of quark indices and which therefore have no counterparts in the original space; this is referred to as the unphysical subspace.

There are a variety of possible ways to ensure that the 
physics of the original quark problem is preserved under the mapping to baryons. We follow the approach of Belyaev and Zelevinsky, where operators in the original space are mapped onto operators in the new space so as to preserve their (anti)commutation relations. Implementation of this prescription guarantees that all the physics of the original system is exactly preserved by the mapping within the physical subspace.

For a mapping to be of practical use, the unphysical states must lie high in energy relative to physical states. Otherwise, it will be difficult to disentangle the physical states of interest from those that are unphysical, particularly in the presence of variational approximations. This places stringent limitations on the kinds of mappings that should be considered for practical applications.

For example, it is straightforward to write down a mapping of colorless particle-hole $(p-h)$ or one-body quark operators that preserves their commutation relations:

$$
\sum_{1} q_{1 a}^{\dagger} q_{1 b} \rightarrow \frac{1}{2} \sum_{123 c d} \Lambda_{1 a 2 c 3 d}^{\dagger} \Lambda_{1 b 2 c 3 d} .
$$

Since any of the colorless operators of the type (2.2)-(2.4) can be rewritten in terms of colorless particle-hole quark operators, it would seem that we could simply apply (2.8) and achieve our goal. This is unfortunately not the case. Since a particle-hole operator does not involve more than one creation and one annihilation operator, it cannot (by itself) incorporate any information on the quark Pauli principle. As a consequence, the spectrum that would result from a pure p-h mapping (for fermion systems) would invariably have unphysical states lying below the physical states of interest. To incorporate quark Pauli effects (in a physically useful way), we must map directly the multiquark creation and annihilation operators that appear in the Hamiltonian.

In what follows we adopt the Dyson approach, which leads to a baryon Hamiltonian that is non-Hermitian but finite. The non-Hermiticity is a direct reflection of quark Pauli effects. A novel feature of our analysis is that, in contrast to earlier work, we focus on the mapping of colorless operators. This removes some of the ambiguities that arise when the mapping is carried out more generally. It also leads to a mapping that is tailored to physical applications in which a truncation to colorless triplets must be implemented.

\section{B. Mapping of colorless one-body operators}

We begin with a discussion of the colorless one-body operator $A_{a b}$ of (2.2). To map this operator, we can make direct use of earlier results. Namely, using (2.8), we can express its baryon image as

$$
A_{a b}=\sum_{1} q_{1 a}^{\dagger} q_{1 b} \rightarrow \frac{1}{2} \sum_{123} \sum_{c d} \Lambda_{1 a 2 c 3 d}^{\dagger} \Lambda_{1 b 2 c 3 d} .
$$

At this point, the image of $A_{a b}$ is expressed in terms of baryons with color. We know, however, that it is possible to describe the relevant physics in a basis of colorless baryons only. Towards this end, it is useful to carry out a truncation to colorless baryons; this can be done using the prescription spelled out by PEDR. The basic idea is to carry out a color-SU(3) coupling and to isolate the piece that is fully antisymmetric in color and fully symmetric in the other noncolor indices. A simple way to implement this is through the replacements

$$
\begin{aligned}
& \Lambda_{1 i 2 j 3 k}^{\dagger} \rightarrow \epsilon_{123} \Lambda_{i j k}^{\dagger}, \\
& \Lambda_{1 i 2 j 3 k} \rightarrow \epsilon_{123} \Lambda_{i j k} .
\end{aligned}
$$

The operators $\Lambda_{i j k}^{\dagger}$ and $\Lambda_{i j k}$ introduced here are fully symmetric under the interchange of their indices. Furthermore, they satisfy the anticommutation relation

$$
\left\{\Lambda_{i j k}, \Lambda_{l m n}^{\dagger}\right\}=\frac{1}{6} S(i j k, l m n)
$$

where

$$
\begin{aligned}
S(i j k, l m n)= & \delta_{i l} \delta_{j m} \delta_{k n}+\delta_{i m} \delta_{j n} \delta_{k l}+\delta_{i n} \delta_{j l} \delta_{k m} \\
& +\delta_{i l} \delta_{j n} \delta_{k m}+\delta_{i m} \delta_{j l} \delta_{k n}+\delta_{i n} \delta_{j m} \delta_{k l}
\end{aligned}
$$

Inserting (2.10) into (2.9) and then carrying out an explicit sum over the color indices $\left(\sum_{123} \epsilon_{123}^{2}=6\right)$, we arrive at the following result:

$$
A_{a b} \rightarrow 3 \sum_{c d} \Lambda_{a c d}^{\dagger} \Lambda_{b c d}
$$

As an example, consider a mapping of the quark number operator $\hat{N}_{Q}=\sum_{1 a} q_{1 a}^{\dagger} q_{1 a}$. Applying (2.13), we obtain the expected result

$$
\hat{N}_{Q} \rightarrow 3 \sum_{a b c} \Lambda_{a b c}^{\dagger} \Lambda_{a b c}=3 \hat{N}_{B}
$$

where $\hat{N}_{B}$ is the number operator for colorless baryons.

\section{Mapping of colorless three-body operators}

Next we consider the colorless three-body operator $C_{a b c d e f}$ appearing in (2.4). Here, too, we can directly use the mapping given by Nadjakov and Meyer, since the set of one-body operators plus the set of three-quark creation and annihilation operators close under commutation. The non-Hermitian mappings of three-quark creation and annihilation operators required to preserve this commutation algebra are $[4,5]$

and

$$
q_{1 i} q_{2 j} q_{3 k} \rightarrow \Lambda_{3 k 2 j 1 i}
$$

$$
\begin{aligned}
q_{1 i}^{\dagger} q_{2 j}^{\dagger} q_{3 k}^{\dagger} \rightarrow & \Lambda_{1 i 2 j 3 k}^{\dagger}+\frac{1}{2} \sum_{456, l m n}\left(\Lambda_{4 l 5 m 1 i}^{\dagger} \Lambda_{2 j 3 k 6 n}^{\dagger}+\Lambda_{4 l 5 m 2 j}^{\dagger} \Lambda_{3 k 1 i 6 n}^{\dagger}+\Lambda_{4 l 5 m 3 k}^{\dagger} \Lambda_{1 i 2 j 6 n}^{\dagger}\right) \Lambda_{4 l 5 m 6 n} \\
& +\frac{1}{8} \sum_{456789, l m n o p q} \Lambda_{4 l 5 m 1 i}^{\dagger} \Lambda_{6 n 7 o 2 j}^{\dagger} \Lambda_{8 p 9 q 3 k}^{\dagger} \Lambda_{4 l 6 n 8 p} \Lambda_{5 m 7 o 9 q}
\end{aligned}
$$


Applying (2.15)-(2.16) to the colorless three-body operator $C_{a b c d e f}$ and carrying out a subsequent truncation to colorless triplets, we obtain the following result:

$$
C_{a b c d e f} \rightarrow C_{a b c d e f}^{n h}=36 \Lambda_{a b c}^{\dagger} \Lambda_{d e f}+36 \sum_{g h i}\left(\Lambda_{g h a}^{\dagger} \Lambda_{b c i}^{\dagger}+\Lambda_{g h b}^{\dagger} \Lambda_{c a i}^{\dagger}+\Lambda_{g h c}^{\dagger} \Lambda_{a b i}^{\dagger}\right) \Lambda_{g h i} \Lambda_{d e f}
$$

A superscript $n h$ has been included to indicate that this is a non-Hermitian baryon image.

Note that a colorless three-quark interaction maps onto colorless one-body plus two-body interactions only. The three-body piece cancels exactly in the truncation to colorless baryons, because of properties of the $\epsilon_{123}$ factors.

\section{Mapping of colorless two-body operators}

Finally we turn to the two-body operator, for which we cannot directly use the results of earlier work. Meyer proposed a possible non-Hermitian baryon image for two-body operators, based on the use of the Usui operator. However, as noted earlier, this mapping does not seem to work when truncated to colorless triplets and applied to the test model we present later. Nevertheless, there is a suggestion in her work as to how to build a proper mapping for colorless two-body operators, which we now exploit. At the end, we make some remarks as to why her results are not applicable.

As we saw in the previous subsection, it is possible to map a colorless three-body operator in non-Hermitian form. Since the mapping (2.17) followed from an exact preservation of commutation relations, it is certainly legitimate. But there is another way to map a colorless three-body operator that is equally legitimate and which leads to a Hermitian form.

The three-body operator $C_{a b c d e f}$ can be rewritten in terms of the colorless one-body operators $A_{i j}$ of $(2.2)$ as follows:

$$
\begin{aligned}
C_{a b c d e f}= & A_{a d} A_{b e} A_{c f}+A_{a d} A_{b f} A_{c e}+A_{a e} A_{b d} A_{c f}+A_{a e} A_{b f} A_{c d}+A_{a f} A_{b d} A_{c e}+A_{a f} A_{b e} A_{c d} \\
& -\delta_{b d}\left(A_{a e} A_{c f}+A_{a f} A_{c e}\right)-\delta_{b e}\left(A_{a d} A_{c f}+A_{a f} A_{c d}\right)-\delta_{b f}\left(A_{a d} A_{c e}+A_{a e} A_{c d}\right) \\
& -2 \delta_{c d}\left(A_{a e} A_{b f}+A_{a f} A_{b e}\right)-2 \delta_{c e}\left(A_{a d} A_{b f}+A_{a f} A_{b d}\right)-2 \delta_{c f}\left(A_{a d} A_{b e}+A_{a e} A_{b d}\right) \\
& +2\left(\delta_{b c, e f} A_{a d}+\delta_{b c, d f} A_{a e}+\delta_{b c, d e} A_{a f}\right)
\end{aligned}
$$

Applying the mapping of colorless one-body operators (2.13) and focusing on the one-and two-body pieces, we obtain, for the Hermitian image,

$$
C_{a b c d e f} \rightarrow C_{a b c d e f}^{h}=36 \Lambda_{a b c}^{\dagger} \Lambda_{d e f}-36 \sum_{g h i}\left(\Lambda_{g h a}^{\dagger} \Lambda_{b c i}^{\dagger}+\Lambda_{g h b}^{\dagger} \Lambda_{c a i}^{\dagger}+\Lambda_{g h c}^{\dagger} \Lambda_{a b i}^{\dagger}\right)\left(\Lambda_{d g h} \Lambda_{e f i}+\Lambda_{e g h} \Lambda_{d f i}+\Lambda_{f g h} \Lambda_{d e i}\right)
$$

Note that the several $\delta$-function terms in (2.18) do not survive after the mapping. They are exactly canceled by other terms that arise when the baryon image is put in normal order.

The one-body piece of (2.19) is identical to that given in (2.17). The two-body part, however, is not. One is Hermitian and the other non-Hermitian. However, both are formally justified and thus must be equivalent in the physical subspace. It is easy to show that either of the forms of the two-baryon image of $C_{a b c d e f}$ can be transformed into the other by performing the following replacement on the two annihilation operators:

$$
\begin{aligned}
\Lambda_{a b c} \Lambda_{d e f} \rightarrow & -\frac{1}{3}\left(\Lambda_{a b d} \Lambda_{c e f}+\Lambda_{a b e} \Lambda_{d c f}+\Lambda_{a b f} \Lambda_{d e c}+\Lambda_{a d c} \Lambda_{b e f}+\Lambda_{a e c} \Lambda_{d b f}+\Lambda_{a f c} \Lambda_{d e b}\right. \\
& \left.+\Lambda_{d b c} \Lambda_{a e f}+\Lambda_{e b c} \Lambda_{d a f}+\Lambda_{f b c} \Lambda_{d e a}\right)
\end{aligned}
$$

These observations suggest a procedure for mapping a colorless two-body interaction. Namely, we first transform it to colorless $\mathrm{p}$ - $\mathrm{h}$ form, then map it using the well-known (and formally justified) colorless p-h mapping (2.13) and finally transform its two-body part to a non-Hermitian form by carrying out the replacement $(2.20)$.

We now apply this prescription to the colorless two-quark operator $B_{a b c d}$ of (2.3). Transforming it to p-h form leads to the result

$$
B_{a b c d}=A_{a c} A_{b d}+A_{a d} A_{b c}-\delta_{b d} A_{a c}-\delta_{b c} A_{a d} .
$$

Mapping this operator in colorless $\mathrm{p}-\mathrm{h}$ form and writing the result in normal order gives

$$
B_{a b c d} \rightarrow B_{a b c d}^{h}=12 \sum_{f} \Lambda_{a b f}^{\dagger} \Lambda_{c d f}-9 \sum_{e f g h} \Lambda_{a e f}^{\dagger} \Lambda_{b g h}^{\dagger}\left(\Lambda_{c e f} \Lambda_{d g h}+\Lambda_{d e f} \Lambda_{c g h}\right) .
$$

Finally, when we impose the replacement $(2.20)$ on the two-baryon piece, we arrive at

$$
B_{a b c d} \rightarrow B_{a b c d}^{n h}=12 \sum_{e} \Lambda_{a b e}^{\dagger} \Lambda_{c d e}+9 \sum_{e f g h} \Lambda_{a e f}^{\dagger} \Lambda_{b g h}^{\dagger}\left(\Lambda_{c d e} \Lambda_{f g h}+\Lambda_{e f g} \Lambda_{c d h}\right)
$$


This result differs from the one that would arise from Meyer's mapping supplemented by a truncation to colorless baryons, reflecting our consistent treatment of color.

In fact, the non-Hermitian mapping of two-body operators proposed by Meyer can be derived in much the same way by considering a general three-body interaction rather than a colorless one. Such a procedure, however, is not unique, since a general three-body operator can be recast as a product of $\mathrm{p}$-h operators in different ways. Explicit consideration of colorless operators removes this ambiguity. The end result is a prescription for transforming from Hermitian to non-Hermitian images that properly incorporates color and is thus meaningful when implementing a truncation to color singlets.

The essential results of this section, proper nonHermitian baryon images of colorless one-, two-, and three-quark operators, are contained in Eqs. (2.13), (2.23), and (2.17), respectively. Although the mapping of two-body operators was not derived by explicit consideration of commutation relations, we have confirmed that this set of baryon images does indeed preserve the com- mutator $\left[B_{a b c d}, E_{e f g}\right]$, where $E_{\text {efg }}=\sum_{123} \epsilon_{123} q_{1 e}^{\dagger} q_{2 f}^{\dagger} q_{3 g}^{\dagger}$. We should also emphasize here that this set of mapping equations can be applied to any colorless constituent quark Hamiltonian written in uncoupled form.

\section{E. Physical content of the replacement procedure}

Some understanding of the replacement procedure proposed to generate a non-Hermitian two-body image from a Hermitian one can be obtained by studying the mapping of a colorless six-quark state,

$$
|a b c, d e f\rangle_{Q}=\frac{1}{36} \sum_{123456} \epsilon_{123} \epsilon_{456} q_{1 a}^{\dagger} q_{2 b}^{\dagger} q_{3 c}^{\dagger} q_{4 d}^{\dagger} q_{5 e}^{\dagger} q_{6 f}^{\dagger}|0\rangle_{Q}
$$

where the subscript $Q$ indicates a state in the original quark space. Mapping this state with (2.16) and imposing a truncation to colorless baryons leads to the result

$$
\begin{aligned}
|a b c, d e f\rangle_{Q} \rightarrow & \Lambda_{a b c}^{\dagger} \Lambda_{d e f}^{\dagger}|0\rangle_{B}+\frac{1}{3}\left\{\Lambda_{a d e}^{\dagger} \Lambda_{b c f}^{\dagger}+\Lambda_{a e f}^{\dagger} \Lambda_{b c d}^{\dagger}+\Lambda_{a d f}^{\dagger} \Lambda_{b c e}^{\dagger}\right. \\
& \left.+\Lambda_{b d e}^{\dagger} \Lambda_{a c f}^{\dagger}+\Lambda_{b e f}^{\dagger} \Lambda_{a c d}^{\dagger}+\Lambda_{b d f}^{\dagger} \Lambda_{a c e}^{\dagger}+\Lambda_{c d e}^{\dagger} \Lambda_{a b f}^{\dagger}+\Lambda_{c e f}^{\dagger} \Lambda_{a b d}^{\dagger}+\Lambda_{c d f}^{\dagger} \Lambda_{a b e}^{\dagger}\right\}|0\rangle_{B}
\end{aligned}
$$

The physical state not only involves the direct twobaryon component but also a sum over all nine possible interchanges of the indices of one baryon with those of the other (with an overall factor of $\frac{1}{3}$ ). It is not difficult to confirm that when we act either with the operator $\Lambda_{a b c} \Lambda_{d e f}$ on this physical state or with the replacement form given in (2.20) we arrive at exactly the same result. Thus the proposed replacement indeed satisfies the desired criterion that it produces the same results within the physical subspace. It is also interesting to note the correspondence between the direct and exchange pieces of (2.25) with the left and right hand sides of (2.20).

\section{THREE-COLOR LIPKIN MODEL}

The three-color Lipkin model is based on the wellknown Lipkin model [10], which can be solved analytically and has been used extensively in nuclear physics for testing many-body approximation methods. Since many of the characteristics of the three-color Lipkin model are already in the original one, we review it briefly here.

The Lipkin model has two levels, each one $\Omega$-fold degenerate, separated by an energy $\Delta$. It is assumed that in the unperturbed ground state $N=\Omega$ particles occupy all the single-particle states in the lower level. The fermion creation and annihilation operators of the model are written as $q_{\sigma p}^{\dagger}$ and $q_{\sigma p}$, respectively. Here, $\sigma$ is a quantum label which characterizes whether the particle is in the lower level, $\sigma=-$, or in the upper one, $\sigma=+$, and $p$ distinguishes which of the $\Omega$ degenerate states of that level the particle occupies. The Hamiltonian of the model includes, in addition to the one-body term, a two- body interaction that scatters pairs of particles among the two levels without changing the $p$ values.

This model can be solved exactly by using group theoretical techniques. It is well known that the set of all possible bilinear products formed from a finite set of creation and annihilation operators constitutes a Lie algebra. In the case of the Lipkin model, there are $(2 \Omega)^{2}$ such bilinear products of creation and annihilation operators (generators), and so the relevant Lie algebra is $U(2 \Omega)$. These generators will be denoted by $K_{\sigma^{\prime} p^{\prime}}^{\sigma p}=q_{\sigma p}^{\dagger} q_{\sigma^{\prime} p^{\prime}}$. Since we are dealing with fermions, all the states of the system belong to the irrep $\left[1^{\Omega}\right]$ of the $U(2 \Omega)$ dynamical group. The structure of the problem suggests the decomposition

$$
\mathrm{U}(2 \Omega) \supset \mathrm{U}(\Omega) \otimes \mathrm{U}(2),
$$

where the $\Omega^{2}$ operators $K_{p^{\prime}}^{p}=\sum_{\sigma} K_{\sigma p^{\prime}}^{\sigma p}$ generate the $\mathrm{U}(\Omega)$ algebra, while the $2^{2}$ objects $K_{\sigma^{\prime}}^{\sigma}=\sum_{p} K_{\sigma^{\prime} p}^{\sigma p}$ are the generators of the $\mathrm{U}(2)$ algebra, and commute with the $K_{p^{\prime}}^{p}$. One can easily verify that the Lipkin Hamiltonian can be written entirely in terms of the $U(2)$ generators and thus that all the states belong to a definite irreducible representation of $\mathrm{U}(2)$ [or $\mathrm{SU}(2)$ ]. This in turn implies that the Hamiltonian matrix can be analytically evaluated using the well-known angular momentum algebra $\mathrm{SU}(2)$.

The three-color Lipkin model has a much richer algebraic structure and analytic solutions are correspondingly more difficult to derive. The model involves three sets (one for each color) of standard two-level Lipkin models. Again the lower levels are assumed to be completely filled in the unperturbed ground state, which in 
this case contains $N=3 \Omega$ particles. The creation and annihilation operators now include a label $i$ that represents the color quantum number and are thus written as $q_{i \sigma p}^{\dagger}$ and $q_{i \sigma p}$, respectively. The model Hamiltonian now includes one-body, two-body, and three-body interactions, which scatter particles coherently among the levels, without changing the $p$ values and maintaining all states colorless:

$$
\begin{gathered}
H=H_{1}+H_{2}+H_{3} \\
H_{1}=\frac{\Delta}{2} \sum_{1 p}\left(q_{1+p}^{\dagger} q_{1+p}-q_{1-p}^{\dagger} q_{1-p}\right) \\
H_{2}=-\frac{\chi_{2}}{\Omega} \sum_{12345, p_{1} p_{2}} \epsilon_{123} \epsilon_{145}\left\{q_{2+p_{1}}^{\dagger} q_{3+p_{2}}^{\dagger} q_{5-p_{2}} q_{4-p_{1}}+q_{4-p_{1}}^{\dagger} q_{5-p_{2}}^{\dagger} q_{3+p_{2}} q_{2+p_{1}}\right\}
\end{gathered}
$$

and

$$
H_{3}=-\frac{\chi_{3}}{\Omega^{2}} \sum_{123456, p_{1} p_{2} p_{3}} \epsilon_{123} \epsilon_{456}\left\{q_{1+p_{1}}^{\dagger} q_{2+p_{2}}^{\dagger} q_{3+p_{3}}^{\dagger} q_{6-p_{3}} q_{5-p_{2}} q_{4-p_{1}}+q_{4-p_{1}}^{\dagger} q_{5-p_{2}}^{\dagger} q_{6-p_{3}}^{\dagger} q_{3+p_{3}} q_{2+p_{2}} q_{1+p_{1}}\right\} .
$$

In this case there are $(6 \Omega)^{2}$ generators $K_{k \sigma^{\prime} p^{\prime}}^{i \sigma p}=q_{i \sigma p}^{\dagger} q_{k \sigma^{\prime} p^{\prime}}$, leading to the Lie algebra $\mathrm{U}(6 \Omega)$, and the structure of the model suggests that we carry out the classification of states in terms of the chain

$$
\mathrm{U}(6 \Omega) \supset \mathrm{U}(\Omega) \otimes \mathrm{U}(6) \supset \mathrm{U}(\Omega) \otimes \mathrm{U}(3) \otimes \mathrm{U}(2)
$$

The $\Omega^{2}$ operators $K_{p^{\prime}}^{p}=\sum_{i \sigma} K_{i \sigma p^{\prime}}^{i \sigma p}$ generate $\mathrm{U}(\Omega)$, while the $6^{2}$ objects $K_{k \sigma^{\prime}}^{i \sigma}=\sum_{p} K_{k \sigma^{\prime} p}^{i \sigma p}$ are the U(6) generators, in terms of which we shall rewrite the Hamiltonian (3.2)-(3.5) below. We can further decompose U(6) by contracting again to the $2^{2}$ operators $K_{\sigma^{\prime}}^{\sigma}=\sum_{i} K_{i \sigma^{\prime}}^{i \sigma}$, which generate $\mathrm{U}(2)$, or to the $3^{2}$ generators $K_{k}^{i}=\sum_{\sigma} K_{k \sigma}^{i \sigma}$ corresponding to $\mathrm{U}(3)$. The latter group is indeed necessary in the classification, since all physically admissible states should be colorless; i.e., they should belong to the $(\Omega, \Omega, \Omega) \mathrm{U}(3)$ representation [which corresponds to the $(\lambda, \mu)=(0,0)$ scalar representation in Elliott's SU(3) notation]. The situation is more complex than in the standard Lipkin model, however, since different $U(6)$ representations can contain these states and, moreover, for each of them several $U(2)$ representations are connected by the Hamiltonian.

From (3.2)-(3.5), we see that the model involves three parameters, one each for the one-, two-, and three-body interactions. As mentioned before, it may be rewritten in terms of the $U(6)$ generators as

$$
\begin{gathered}
H_{1}=\Delta J_{z} \\
H_{2}=-\frac{\chi_{2}}{\Omega}\left(J_{+}^{2}+J_{-}^{2}\right)+\frac{\chi_{2}}{\Omega} \sum_{i k}\left(K_{k-}^{i+} K_{i-}^{k+}+K_{k+}^{i-} K_{i+}^{k-}\right)
\end{gathered}
$$

and

$$
H_{3}=-\frac{\chi_{3}}{\Omega^{2}}\left(J_{+}^{3}+J_{-}^{3}\right)+\frac{3 \chi_{3}}{\Omega^{2}}\left(J_{+} \sum_{i k} K_{k-}^{i+} K_{i-}^{k+}+J_{-} \sum_{i k} K_{k+}^{i-} K_{i+}^{k-}\right)-\frac{2 \chi_{3}}{\Omega^{2}} \sum_{i l k}\left(K_{l-}^{i+} K_{k-}^{l+} K_{i-}^{k+}+K_{l+}^{i-} K_{k+}^{l-} K_{i+}^{k-}\right)
$$

where $J_{z}, J_{+}$, and $J_{-}$, defined by $J_{z} \equiv \frac{1}{2}\left(K_{+}^{+}-K_{-}^{-}\right), J_{+} \equiv K_{-}^{+}$, and $J_{-} \equiv K_{+}^{-}$, are the $\mathrm{SU}(2)$ subgroup generators, which together with the number operator $\hat{N}=\sum_{i \sigma p} K_{i \sigma p}^{i \sigma p}$ comprise the U(2) group in (3.6). The other operators in (3.8) and (3.9), namely, $\sum_{i k} K_{k-}^{i+} K_{i-}^{k+}$ and $\sum_{i l k} K_{l-}^{i+} K_{k-}^{l+} K_{i-}^{k+}$, together with their Hermitian conjugates, clearly lie outside $\mathrm{SU}(2)$. Using their commutation relations with the $\mathrm{SU}(2)$ generators, we readily conclude that they behave as rank 2 and rank 3 tensors $T_{m}^{(\ell)}=T_{2}^{(2)}$ and $T_{3}^{(3)}$ in $\mathrm{SU}(2)$, respectively.

Using this notation, the model Hamiltonian acquires the simpler form

$$
\begin{gathered}
H_{1}=\Delta J_{z} \\
H_{2}=-\frac{\chi_{2}}{\Omega}\left(J_{+}^{2}+J_{-}^{2}\right)+\frac{\chi_{2}}{\Omega}\left(T_{2}^{(2)}+T_{-2}^{(2)}\right)
\end{gathered}
$$

and

$$
H_{3}=-\frac{\chi_{3}}{\Omega^{2}}\left(J_{+}^{3}+J_{-}^{3}\right)+\frac{3 \chi_{3}}{\Omega^{2}}\left(J_{+} T_{2}^{(2)}+J_{-} T_{-2}^{(2)}\right)-\frac{2 \chi_{3}}{\Omega^{2}}\left(T_{3}^{(3)}-T_{-3}^{(3)}\right)
$$


to which we shall refer henceforth.

Since we are dealing with a system of fermions, the states of the model belong to the $\left[1^{3 \Omega}\right]$ representation of $\mathrm{U}(6 \Omega)$, while the $\mathrm{U}(\Omega)$ and $\mathrm{U}(6)$ representations are complementary; i.e., once the $\mathrm{U}(6)$ representation is determined, the $U(\Omega)$ representation is fixed. Since the $U(3)$ representations are the colorless ones mentioned before, it should be clear that the basis states can be unambiguously denoted by

$$
\left|\left[h_{1}, h_{2}, h_{3}, h_{4}, h_{5}, h_{6}\right], \alpha j m\right\rangle,
$$

where $\left[h_{1}, \ldots, h_{6}\right]$ labels the $\mathrm{U}(6)$ representations, $j m$ are the $\mathrm{SU}(2) \supset \mathrm{SO}(2)$ quantum numbers, and $\alpha$ is an extra label that may be needed to distinguish either repeated $\mathrm{U}(6)$ irreducible representations (irreps) within the same $\mathrm{U}(6 \Omega)$ representation, repeated $j$ 's within the same $\mathrm{U}(6)$ representation, or any other necessary quantum numbers. For example, the unperturbed ground state of the model, defined as the state for which all $\sigma=-$ levels are filled, corresponds to

$$
|G\rangle=\left|[\Omega, \Omega, \Omega, 0,0,0], j=\frac{3 \Omega}{2}, m=-\frac{3 \Omega}{2}\right\rangle \text {. }
$$

The state $|G\rangle$ has three particles with $p=1$, three with $p=2, \ldots$, and three with $p=\Omega$. Defining $P=\sum_{i} p_{i}$, we see that the state $|G\rangle$ has a unique $P$ value of $3 \Omega(\Omega+1) / 2$.

In general, different $\mathrm{U}(6)$ irreducible representations appear which contain the $\mathrm{U}(3)$ colorless irreps in the reduction $U(6) \supset U(3) \otimes U(2)$ of (3.6). Since the Hamiltonian (3.10)-(3.12) is built solely in terms of $U(6)$ generators, the energy matrix will separate into blocks, each corresponding to a definite $\mathrm{U}(6)$ irrep.

For $\Omega=1$, the group analysis is particularly simple. There is only a single $U(6)$ irrep, $(1,1,1,0,0,0)$, which contains the $(1,1,1) \otimes(j=3 / 2)$ and the $(2,1,0) \otimes(j=1 / 2)$ $\mathrm{U}(3) \otimes \mathrm{SU}(2)$ irreps. However, only the first one belongs to our (colorless) space. Furthermore, all states have $P=3$.

For $\Omega=2$ the situation is more complex in several respects. First, as we shall enumerate shortly, there are several possible $\mathrm{U}(6)$ irreps that contain colorless states. All contain states with $P=9$, while several also contain states with other $P$ values. These other states, however, can be generated from the corresponding $P=9$ states by the $\mathrm{U}(\Omega)$ raising and lowering operators $K_{p}^{p^{\prime}}$, with $p \neq p^{\prime}$, and are thus degenerate in energy with them. For this reason, we need only consider the states with $P=9$ to fully exhaust the spectrum. In Table I, we display the four possible $\Omega=2 \quad \mathrm{U}(6)$ irreps that contain colorless $P=9$ states and also indicate the associated angular momenta. A similar analysis can also be carried out for higher $\Omega$ values.

TABLE I. Colorless states and group labels for $\Omega=2$.

\begin{tabular}{ccc}
\hline $\mathrm{U}(6)$ labels & $\mathrm{SU}(2)$ label & Degeneracy \\
\hline$[2,2,2,0,0,0]$ & $j=1,3$ & 10 \\
{$[2,2,1,1,0,0]$} & $j=0,2$ & 6 \\
{$[2,1,1,1,1,0]$} & $j=1$ & 3 \\
{$[1,1,1,1,1,1]$} & $j=0$ & 1 \\
\hline \hline
\end{tabular}

In the following section we test our mapping procedure for the case of $\Omega=2$. Here, we illustrate the algebraic evaluation of matrix elements for the $[2,2,2,0,0,0] \Omega=2$ submatrix, for which we will need to consider states with $j=3$ and $j=1$ (see Table I). Analogous calculations have also been done for the other states and are included in the results that we present.

Before proceeding, we write down the commutation relation for the $U(6)$ generators, which will be used extensively in our analysis:

$$
\left[K_{n \sigma_{2}}^{i \sigma_{1}}, K_{l \sigma_{4}}^{k \sigma_{3}}\right]=\delta_{k n} \delta_{\sigma_{2} \sigma_{3}} K_{l \sigma_{4}}^{i \sigma_{1}}-\delta_{i l} \delta_{\sigma_{1} \sigma_{4}} K_{n \sigma_{2}}^{k \sigma_{3}}
$$

We simplify the notation and write the $[2,2,2,0,0,0]$ set of states as $|j, m\rangle$. We also note that the $\mathrm{U}(3)$ operators $K_{k}^{i}=\sum_{\sigma} K_{k \sigma}^{i \sigma}$ act as color raising or lowering operators if $k \neq i$. Thus, acting on a colorless state, they give zero unless $k=i$, i.e.,

$$
\left(K_{k+}^{i+}+K_{k-}^{i-}\right)|j, m\rangle=\delta_{k i} A|j, m\rangle .
$$

To find the value of $A$, we note that

$$
\sum_{k}\left(K_{k+}^{k+}+K_{k-}^{k-}\right)|j, m\rangle=N|j, m\rangle=6|j, m\rangle,
$$

for $\Omega=2$. Since all colors should be equally represented in a colorless state, we arrive at the useful relation

$$
\left(K_{k+}^{i+}+K_{k-}^{i-}\right)|j, m\rangle=2 \delta_{k i}|j, m\rangle \text {. }
$$

The basic idea of our analysis is to consider the action of the operators $T_{2}^{(2)}$ and $T_{3}^{(3)}$ on the states $|j, m\rangle=$ $|3,-3\rangle$ and $|1,-1\rangle$, namely,

$$
\begin{gathered}
T_{2}^{(2)}|3,-3\rangle=a_{3,0}|3,-1\rangle+a_{3,-2}|1,-1\rangle, \\
T_{3}^{(3)}|3,-3\rangle=b_{3,0}|3,0\rangle+b_{3,-2}|1,0\rangle \\
T_{2}^{(2)}|1,-1\rangle=a_{1,0}|1,+1\rangle+a_{1,2}|3,+1\rangle,
\end{gathered}
$$

and

$$
T_{3}^{(3)}|1,-1\rangle=b_{1,2}|3,+2\rangle \text {. }
$$

We use a notation whereby the first index in the expansion coefficients denotes the $\mathrm{SU}(2)$ label of the initial state and the second gives the increment needed to obtain the $\mathrm{SU}(2)$ label of the final state.

If we can determine all of the independent $a$ and $b$ expansion coefficients in (3.17)-(3.20), we will have effectively solved the problem. We can then use the WignerEckart theorem to determine all the relevant reduced matrix elements of $T^{(2)}$ and $T^{(3)}$ and from them determine all of the matrix elements of the Hamiltonian for any choice of the model parameters. We now outline a simple algebraic procedure for evaluating these expansion coefficients.

We first consider the calculation of the coefficients appearing in (3.17) and (3.18). By using the $\mathrm{U}(6)$ commutation relation (3.15) repeatedly, we find that 


$$
\left[J^{2}, T_{2}^{(2)}\right]=\left(4 J_{z}+2\right) T_{2}^{(2)}+J_{+} \sum_{k i}\left[K_{k-}^{i+}\left(K_{i-}^{k-}-K_{i+}^{k+}\right)+\left(K_{k-}^{i-}-K_{k+}^{i+}\right) K_{i-}^{k+}\right]
$$

which leads to

$$
J^{2} T_{2}^{(2)}|G\rangle=2 T_{2}^{(2)}|G\rangle-2 J_{+}^{2}|G\rangle .
$$

Doing the same for $T_{3}^{(3)}$, we obtain

$$
J^{2} T_{3}^{(3)}|G\rangle=-3 J_{+} T_{2}^{(2)}|G\rangle-3 J_{+}^{3}|G\rangle .
$$

To calculate $a_{3,0}$, we multiply $(3.22)$ by $\langle 3,-1|$ and use (3.18). Remembering also that $|G\rangle=|3,-3\rangle$, we find that

$$
\left\langle 3,-1\left|J^{2} T_{2}^{(2)}\right| 3,-3\right\rangle=2 a_{3,0}-4 \sqrt{15} .
$$

Applying $J^{2}$ to the left and noting that

$$
a_{3,0}=\left\langle 3,-1\left|T_{2}^{(2)}\right| 3,-3\right\rangle,
$$

we obtain

$$
a_{3,0}=-2 \sqrt{\frac{3}{5}}
$$

To calculate $a_{3,-2}$, we need the overlap $\mathcal{N}=\langle G|$ $\left(T_{2}^{(2)}\right)^{\dagger} T_{2}^{(2)}|G\rangle$. A simple calculation using the commutator relation $(3.15)$ gives

$$
\mathcal{N}=60
$$

which leads to

$$
a_{3,-2}=12 \sqrt{\frac{2}{5}} .
$$

There remains an undetermined overall sign for this coefficient, which can be chosen arbitrarily with no change in the final results.

A similar procedure can be used to evaluate $b_{3,0}$ and $b_{3,-2}$. In particular, to obtain $b_{3,0}$, we multiply $(3.23)$ by $\langle 3,0|$, which leads to

$$
b_{3,0}=-\frac{12}{\sqrt{5}} .
$$

Likewise, to obtain $b_{3,-2}$, we multiply $(3.23)$ by $\langle 1,0|$; the result is

$$
b_{3,-2}=-\frac{36}{\sqrt{5}}
$$

Finally, we turn to the last independent coefficient $a_{1,0}$, which appears in (3.19). We must first introduce another tensor operator $T_{4}^{(4)}=\left(T_{2}^{(2)}\right)^{2}$, whose action on the unperturbed ground state $|G\rangle$ can be readily shown to be

$$
J^{2} T_{4}^{(4)}|G\rangle=-4 J_{+}^{2} T_{2}^{(2)}|G\rangle-8 J_{+} T_{3}^{(3)}|G\rangle .
$$

To determine $a_{1,0}$, we multiply $(3.31)$ by $\langle 1,1|$ and apply $J^{2}$ to the left. This leads to

$$
\begin{aligned}
2 a_{3,0}\left\langle 1,1\left|T_{2}^{(2)}\right| 3,-1\right\rangle+ & 2 a_{3,-2} a_{1,0} \\
& =-8 a_{3,-2}-8 \sqrt{2} b_{3,-2} .
\end{aligned}
$$

Using the Wigner-Eckart theorem to relate

$$
\left\langle 1,1\left|T_{2}^{(2)}\right| 3,-1\right\rangle \text { to }\left\langle 1,-1\left|T_{2}^{(2)}\right| 3,-3\right\rangle
$$

and the earlier results for $a_{3,0}, a_{3,-2}$, and $b_{3,-2}$, we find that

$$
a_{1,0}=\frac{42}{5}
$$

From the $a$ and $b$ coefficients evaluated above, we can determine all the remaining coefficients as well as all possible reduced matrix elements of interest by using the Wigner-Eckart theorem; the results are

$$
\left\langle j=3\left|T^{(2)}\right| j=3\right\rangle=-6 \sqrt{\frac{14}{5}},
$$

$$
\left\langle j=1\left\|T^{(2)}\right\| j=3\right\rangle=\left\langle j=3\left\|T^{(2)}\right\| j=1\right\rangle=12 \sqrt{\frac{14}{5}},
$$

$$
\left\langle j=1\left\|T^{(2)}\right\| j=1\right\rangle=\frac{42}{\sqrt{5}},
$$

$$
\begin{gathered}
\left\langle j=3\left\|T^{(3)}\right\| j=3\right\rangle=12 \sqrt{\frac{42}{5}}, \\
\left\langle j=1\left\|T^{(3)}\right\| j=3\right\rangle=\left\langle j=3\left\|T^{(2)}\right\| j=1\right\rangle=72 \sqrt{\frac{7}{15}}, \\
\left\langle j=1\left\|T^{(3)}\right\| j=1\right\rangle=0 .
\end{gathered}
$$

These results coupled with further use of the WignerEckart theorem permit us to determine all matrix elements of the three-color Lipkin Hamiltonian, which because of the small number of basis states can be easily diagonalized.

We have limited our algebraic analysis here to $\Omega=2$, since that is the case for which we carry our mapping tests in the next section, but it is possible to use similar algebraic techniques for larger values of $\Omega$. The method, however, rapidly becomes more complicated for increasing $\Omega$. The reason is that more $j$ values appear for larger $\Omega$ and an iterative procedure is required to determine the action of $T_{2}^{(2)}$ and $T_{3}^{(3)}$ on progressively smaller angular momentum states. As we have seen in the $\Omega=2$ case, each step in the iterative procedure requires the introduction of a new tensor operator, built out of the fundamental ones. We have already succeeded in obtaining algebraic results for $\Omega=3$ using similar methods. Their generalization to arbitrary $\Omega$ is currently being investigated. 


\section{TEST OF MAPPING ON THE THREE-COLOR LIPKIN MODEL}

In this section we apply the colorless baryon mapping developed in Sec. II to the three-color Lipkin model. We carry out the analysis and the resulting comparisons for $\Omega=2$ only, for which the number of baryons is likewise 2 . Diagonalization of the effective triplet Hamiltonian can be done exactly for this case, leading to a direct test of the mapping.

\section{A. Construction of the colorless baryon space}

The colorless states of the model, after carrying out the mapping, are constructed in terms of baryons with quantum numbers $\sigma_{1} p_{1}, \sigma_{2} p_{2}$, and $\sigma_{3} p_{3}$. As in the previous section, two noncolor quantum numbers are needed to specify the state of each of the three quarks represented by the colorless baryon. Since all three quarks have different colors, there is no Pauli restriction on these quantum numbers.

As noted in the previous section, a useful way to characterize states of the model is in terms of the total $P$ value, which for a single baryon is $P=p_{1}+p_{2}+p_{3}$. In Table II, we enumerate the number of distinct colorless one-baryon states for each possible value of $P$, ranging from $P=3-6(=3 \Omega)$.

The two-baryon states of particular interest are those with total $P=P_{1}+P_{2}=9$. There are two ways to achieve $P=9$, either with one triplet having $P=3$ and the other $P=6$, or with one having $P=4$ and the other $P=5$. From Table II, we see that the number of distinct two-triplet states with $P=9$ is $52 ; 16$ have $\left(P_{1}, P_{2}\right)=(3,6)$ and 36 have $\left(P_{1}, P_{2}\right)=(4,5)$. This is significantly larger than the number of $P=9$ states in the original quark model (see Table I), which is 20 .
TABLE II. Number of distinct colorless one-triplet states $\Lambda_{\sigma_{1} p_{1} \sigma_{2} p_{2} \sigma_{3} p_{3}}|0\rangle_{B}$ for a given total $P=p_{1}+p_{2}+p_{3}$.

\begin{tabular}{rc}
\hline$P$ & No. of states \\
\hline 3 & 4 \\
4 & 6 \\
5 & 6 \\
6 & 4 \\
\hline
\end{tabular}

The reason is that the two-triplet space includes both physical and unphysical states. A central theme of our analysis will be to confirm that our non-Hermitian mapping not only reproduces the spectrum of physical states (as obtained in the algebraic analysis of Sec. III) but also pushes up the unphysical states relative to the Hermitian (pure p-h) mapping.

\section{B. Mapping the Hamiltonian}

The general three-color Lipkin Hamiltonian, given by (3.2)-(3.5), can be mapped either in non-Hermitian or Hermitian form. We will be particularly interested in the non-Hermitian mapping, since it is expected to provide a more practical incorporation of quark Pauli effects. However, in what follows, we present both, to see whether our expectations are indeed realized.

The non-Hermitian (nh) mapping is implemented by using (2.13) for the one-quark term, (2.23) for the twoquark interaction, and (2.17) for the three-quark interaction. The resulting effective Hamiltonian $H_{\mathrm{nh}}$ for colorless baryons is given by

$$
H \rightarrow H_{\mathrm{nh}}=T_{\mathrm{nh}}+V_{\mathrm{nh}}
$$

where

$$
\begin{aligned}
T_{\mathrm{nh}}= & \frac{3 \Delta}{2} \sum_{p_{1} p_{2} p_{3} \sigma_{2} \sigma_{3}}\left\{\Lambda_{+p_{1} \sigma_{2} p_{2} \sigma_{3} p_{3}}^{\dagger} \Lambda_{+p_{1} \sigma_{2} p_{2} \sigma_{3} p_{3}}-\Lambda_{-p_{1} \sigma_{2} p_{2} \sigma_{3} p_{3}}^{\dagger} \Lambda_{-p_{1} \sigma_{2} p_{2} \sigma_{3} p_{3}}\right\} \\
& -\frac{12 \chi_{2}}{\Omega} \sum_{p_{1} p_{2} p_{3} \sigma_{3}}\left\{\Lambda_{+p_{1}+p_{2} \sigma_{3} p_{3}}^{\dagger} \Lambda_{-p_{1}-p_{2} \sigma_{3} p_{3}}+\Lambda_{-p_{1}-p_{2} \sigma_{3} p_{3}}^{\dagger} \Lambda_{+p_{1}+p_{2} \sigma_{3} p_{3}}\right\} \\
& -\frac{36 \chi_{3}}{\Omega^{2}} \sum_{p_{1} p_{2} p_{3}}\left\{\Lambda_{+p_{1}+p_{2}+p_{3}}^{\dagger} \Lambda_{-p_{1}-p_{2}-p_{3}}+\Lambda_{-p_{1}-p_{2}-p_{3}}^{\dagger} \Lambda_{+p_{1}+p_{2}+p_{3}}\right\}
\end{aligned}
$$

and

$$
\begin{aligned}
& V_{\mathrm{nh}}=-\frac{9 \chi_{2}}{\Omega} \sum_{p_{1} \rightarrow p_{6} \sigma_{3} \rightarrow \sigma_{6}}\left\{\Lambda_{+p_{1} \sigma_{3} p_{3} \sigma_{4} p_{4}}^{\dagger} \Lambda_{+p_{2} \sigma_{5} p_{5} \sigma_{6} p_{6}}^{\dagger}\left(\Lambda_{-p_{1}-p_{2} \sigma_{3} p_{3}} \Lambda_{\sigma_{4} p_{4} \sigma_{5} p_{5} \sigma_{6} p_{6}}+\Lambda_{\sigma_{3} p_{3} \sigma_{4} p_{4} \sigma_{6} p_{6}} \Lambda_{-p_{1}-p_{2} \sigma_{5} p_{5}}\right)\right. \\
& \left.+\Lambda_{-p_{1} \sigma_{3} p_{3} \sigma_{4} p_{4}}^{\dagger} \Lambda_{-p_{2} \sigma_{5} p_{5} \sigma_{6} p_{6}}^{\dagger}\left(\Lambda_{+p_{1}+p_{2} \sigma_{3} p_{3}} \Lambda_{\sigma_{4} p_{4} \sigma_{5} p_{5} \sigma_{6} p_{6}}+\Lambda_{\sigma_{3} p_{3} \sigma_{4} p_{4} \sigma_{6} p_{6}} \Lambda_{+p_{1}+p_{2} \sigma_{5} p_{5}}\right)\right\} \\
& -\frac{108 \chi_{3}}{\Omega^{2}} \sum_{p_{1} \rightarrow p_{6} \sigma_{4} \rightarrow \sigma_{6}}\left(\Lambda_{+p_{1} \sigma_{4} p_{4} \sigma_{5} p_{5}}^{\dagger} \Lambda_{+p_{2}+p_{3} \sigma_{6} p_{6}}^{\dagger} \Lambda_{\sigma_{4} p_{4} \sigma_{5} p_{5} \sigma_{6} p_{6}} \Lambda_{-p_{1}-p_{2}-p_{3}}\right. \\
& \left.+\Lambda_{-p_{1} \sigma_{4} p_{4} \sigma_{5} p_{5}}^{\dagger} \Lambda_{-p_{2}-p_{3} \sigma_{6} p_{8}}^{\dagger} \Lambda_{\sigma_{4} p_{4} \sigma_{5} p_{5} \sigma_{6} p_{6}} \Lambda_{+p_{1}+p_{2}+p_{3}}\right) \text {. }
\end{aligned}
$$

The Hermitian (h) mapping is implemented by using (2.13) for the one-body term, (2.22) for the two-body term, and (2.19) for the three-body interaction. The final result of this mapping is 


$$
H \rightarrow H_{\mathrm{h}}=T_{\mathrm{h}}+V_{\mathrm{h}}
$$

where

$$
T_{\mathrm{h}}=T_{\mathrm{nh}}
$$

as given by (4.2), and

$$
\begin{aligned}
& V_{\mathrm{h}}=\frac{9 \chi_{2}}{\Omega} \sum_{p_{1} \rightarrow p_{6} \sigma_{3} \rightarrow \sigma_{8}}\left\{\Lambda_{+p_{1} \sigma_{3} m_{3} \sigma_{4} p_{4}}^{\dagger} \Lambda_{+p_{2} \sigma_{5} p_{5} \sigma_{6} p_{6}}^{\dagger}\left(\Lambda_{-p_{1} \sigma_{3} p_{3} \sigma_{4} p_{4}} \Lambda_{-p_{2} \sigma_{5} p_{5} \sigma_{8} p_{8}}+\Lambda_{-p_{2} \sigma_{3} p_{3} \sigma_{4} p_{4}} \Lambda_{-p_{1} \sigma_{5} p_{5} \sigma_{8} p_{6}}\right)\right. \\
& \left.+\Lambda_{-p_{1} \sigma_{3} p_{3} \sigma_{4} p_{4}}^{\dagger} \Lambda_{-p_{2} \sigma_{5} p_{5} \sigma_{8} p_{6}}^{\dagger}\left(\Lambda_{+p_{1} \sigma_{3} p_{3} \sigma_{4} p_{4}} \Lambda_{+p_{2} \sigma_{5} p_{5} \sigma_{6} p_{6}}+\Lambda_{+p_{2} \sigma_{3} p_{3} \sigma_{4} p_{4}} \Lambda_{+p_{1} \sigma_{5} p_{5} \sigma_{6} p_{6}}\right)\right\} \\
& +\frac{108 \chi_{3}}{\Omega^{2}} \sum_{p_{1} \rightarrow p_{6} \sigma_{4} \rightarrow \sigma_{6}}\left\{\Lambda _ { + p _ { 1 } \sigma _ { 4 } p _ { 4 } \sigma _ { 5 } p _ { 5 } } ^ { \dagger } \Lambda _ { + p _ { 2 } + p _ { 3 } \sigma _ { 8 } p _ { 8 } } ^ { \dagger } \left(\Lambda_{-p_{1} \sigma_{4} p_{4} \sigma_{5} p_{5}} \Lambda_{-p_{2}-p_{3} \sigma_{6} p_{6}}\right.\right. \\
& \left.+\Lambda_{-p_{2} \sigma_{4} p_{4} \sigma_{5} p_{5}} \Lambda_{-p_{3}-p_{1} \sigma_{6} p_{6}}+\Lambda_{-p_{3} \sigma_{4} p_{4} \sigma_{5} p_{5}} \Lambda_{-p_{1}-p_{2} \sigma_{6} p_{6}}\right) \\
& +\Lambda_{-p_{1} \sigma_{4} p_{4} \sigma_{5} p_{5}}^{\dagger} \Lambda_{-p_{2}-p_{3} \sigma_{8} p_{8}}^{\dagger}\left(\Lambda_{+p_{1} \sigma_{4} p_{4} \sigma_{5} p_{5}} \Lambda_{+p_{2}+p_{3} \sigma_{8} p_{8}}\right.
\end{aligned}
$$

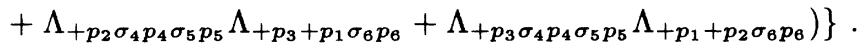

\section{Physical versus unphysical states}

Since one of the aims of this work is to assess the feasibility of "pushing up" unphysical states with respect to physical states by using the non-Hermitian mapping, it is useful to have a criterion for identifying which states are physical and which are unphysical. This can be readily done by introducing a Majorana-like operator [11], analogous to the one used in boson mappings, with the property that its eigenvalues in the physical subspace are zero whereas those in the unphysical subspace are not. There are a variety of such operators. In particular, if we consider any many-body operator in the quark space and map it in both Hermitian and nonHermitian form, the difference between the two resulting triplet operators must give a zero eigenvalue in the physical subspace. Here, as is customary for boson mappings, we focus on the square of the quark number operator, $\hat{N}_{Q}^{2}=\sum_{12 \sigma_{1} \sigma_{2} p_{1} p_{2}} q_{1 \sigma_{1} p_{1}}^{\dagger} q_{1 \sigma_{1} p_{1}} q_{2 \sigma_{2} p_{2}}^{\dagger} q_{2 \sigma_{2} p_{2}}$. Mapping this operator in Hermitian (colorless $\mathrm{p}-\mathrm{h}$ ) form gives

$$
\begin{aligned}
\hat{N}_{\mathrm{h}}^{2}= & 9 \sum_{p_{1} \rightarrow p_{3} \sigma_{1} \rightarrow \sigma_{3}} \Lambda_{\sigma_{1} p_{1} \sigma_{2} p_{2} \sigma_{3} p_{3}}^{\dagger} \Lambda_{\sigma_{1} p_{1} \sigma_{2} p_{2} \sigma_{3} p_{3}} \\
& +9 \sum_{p_{1} \rightarrow p_{6} \sigma_{1} \rightarrow \sigma_{8}} \Lambda_{\sigma_{1} p_{1} \sigma_{2} p_{2} \sigma_{3} p_{3}}^{\dagger} \Lambda_{\sigma_{4} p_{4} \sigma_{5} p_{5} \sigma_{6} p_{8}}^{\dagger} \\
& \times \Lambda_{\sigma_{4} p_{4} \sigma_{5} p_{5} \sigma_{6} p_{8}} \Lambda_{\sigma_{1} p_{1} \sigma_{2} p_{2} \sigma_{3} p_{3}}
\end{aligned}
$$

while implementing the replacement (2.22) leads to the non-Hermitian form

$$
\begin{aligned}
\hat{N}_{\mathrm{nh}}^{2}= & 9 \sum_{p_{1} \rightarrow p_{3} \sigma_{1} \rightarrow \sigma_{3}} \Lambda_{\sigma_{1} p_{1} \sigma_{2} p_{2} \sigma_{3} p_{3}}^{\dagger} \Lambda_{\sigma_{1} p_{1} \sigma_{2} p_{2} \sigma_{3} p_{3}} \\
& -27 \sum_{p_{1} \rightarrow p_{6} \sigma_{1} \rightarrow \sigma_{6}} \Lambda_{\sigma_{1} p_{1} \sigma_{2} p_{2} \sigma_{3} p_{3}}^{\dagger} \Lambda_{\sigma_{4} p_{4} \sigma_{5} p_{5} \sigma_{6} p_{6}}^{\dagger} \\
& \times \Lambda_{\sigma_{1} p_{1} \sigma_{5} p_{5} \sigma_{6} p_{8}} \Lambda_{\sigma_{4} p_{4} \sigma_{2} p_{2} \sigma_{3} p_{3}}
\end{aligned}
$$

An appropriate Majorana operator is

$$
M=\hat{N}_{\mathrm{h}}^{2}-\hat{N}_{\mathrm{nh}}^{2} .
$$

With this choice of sign, the expectation value of $M$ for any unphysical state is positive definite. More specifically, for the three-color Lipkin model with $\Omega=2$ it is precisely +36 for all unphysical states.

\section{Diagonalization of the mapped Hamiltonian and discussion of results}

The mapped Hamiltonian, in either non-Hermitian or Hermitian form, can be readily diagonalized in the full space of colorless two-baryon states for any choice of the strength parameters $\Delta, \chi_{2}$, and $\chi_{3}$. For the nonHermitian mapping, both left and right diagonalization is required to obtain complete information on the eigenvectors. Some representative results are presented in Figs. 1-3. In all three figures, corresponding to three distinct choices of the strength parameters, we present the algebraic results (denoted exact) and the results obtained after both the non-Hermitian (nh) and Hermitian (h) mappings. In the spectra that refer to diagonalization after the mapping, we explicitly distinguish physical from unphysical states, by using the Majorana operator (4.9). Physical states are indicated by solid lines and unphysical states by dashed lines. We use a heavy solid line to denote degenerate (or nearly degenerate) solutions, and indicate to the right the number of physical $(P)$ and unphysical (U) states at that energy. This information can likewise be obtained by judicious use of the Majorana expectation values. Finally, for simplicity, only the relatively low-energy portions of the spectra are shown.

Figure 1 shows our results for the case $\Delta=0$, $\chi_{2}=1$, and $\chi_{3}=0$, namely, for a system dominated by two-quark correlations. There are several points to note. First, both the Hermitian and non-Hermitian mappings exactly reproduce the spectrum of physical states obtained by diagonalization of the original quark model. Second, following the Hermitian mapping, the lowest eigenvalues are indeed unphysical. When the non-Hermitian mapping is used, however, the unphysical states are pushed up in energy, and the lowest four mapped eigenvalues are physical. This is precisely what we had hoped would occur. 


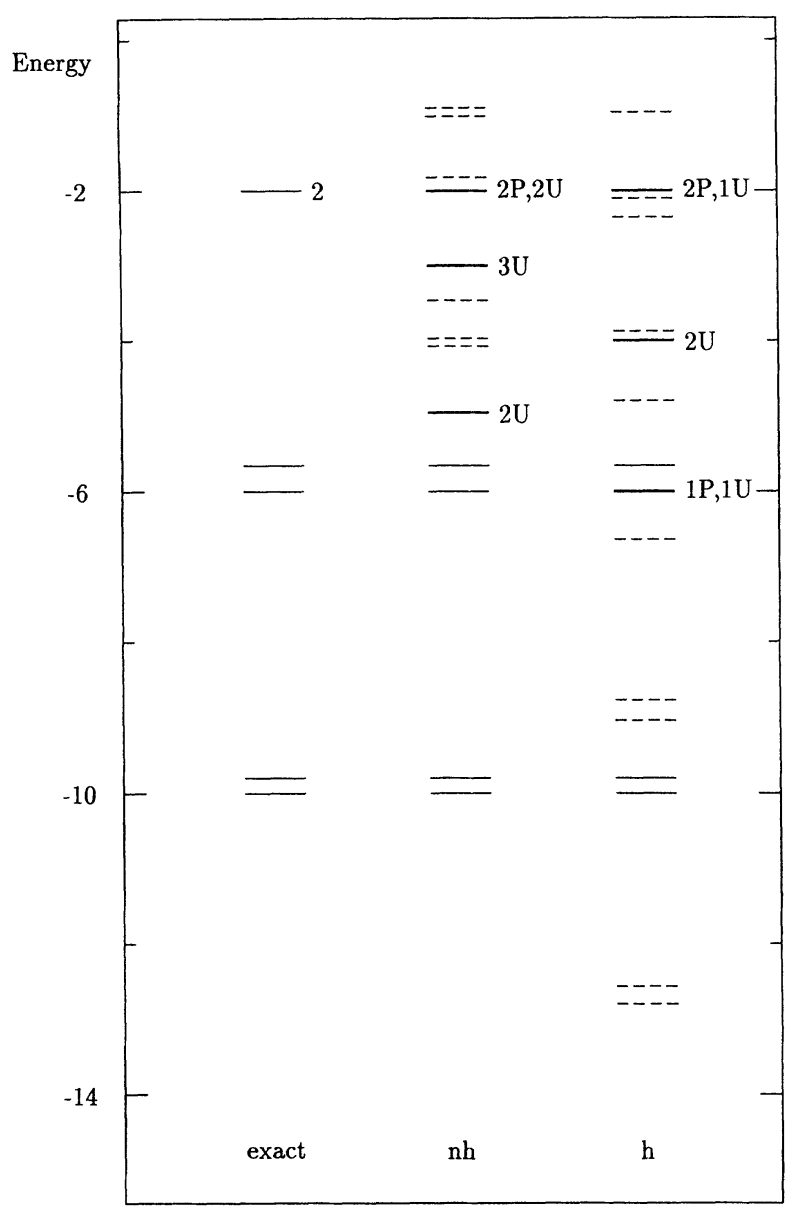

FIG. 1. Calculated spectra of the three-color Lipkin model for $\Omega=2, \Delta=0, \chi_{2}=1$, and $\chi_{3}=0$. Only the levels with $E<0$ are shown. The spectrum denoted exact refers to a diagonalization of the Hamiltonian in the original quark space. Degenerate levels in this spectrum include to the right the degeneracy. The spectra denoted $\mathrm{nh}$ and $\mathrm{h}$ refer to results obtained following non-Hermitian and Hermitian triplet-fermion mappings, respectively. Physical states in the mapped spectra are denoted by solid lines and unphysical states by dashed lines. Heavy solid lines indicate degenerate (or nearly degenerate) solutions; to the right are given the number of physical and unphysical states at that energy.

It is important, however, to see whether this also occurs in the presence of three-quark correlations. Towards that end, we show in Fig. 2 the analogous results obtained for $\Delta=0, \chi_{2}=0$, and $\chi_{3}=1$, namely, for a system dominated by three-quark correlations. And, indeed, the same conclusions apply. Both mappings exactly reproduce the spectrum of physical states. The Hermitian mapping, however, leads to unphysical states very low in energy, whereas the non-Hermitian mapping yields them significantly raised.

Finally, in Fig. 3, we present the results for a scenario in which all three terms in the quark Hamiltonian are active: $\Delta=1, \chi_{2}=1$, and $\chi_{3}=1$. Once again, both mappings reproduce the physical spectrum, while the non-Hermitian mapping pushes up the unphysical states substantially.

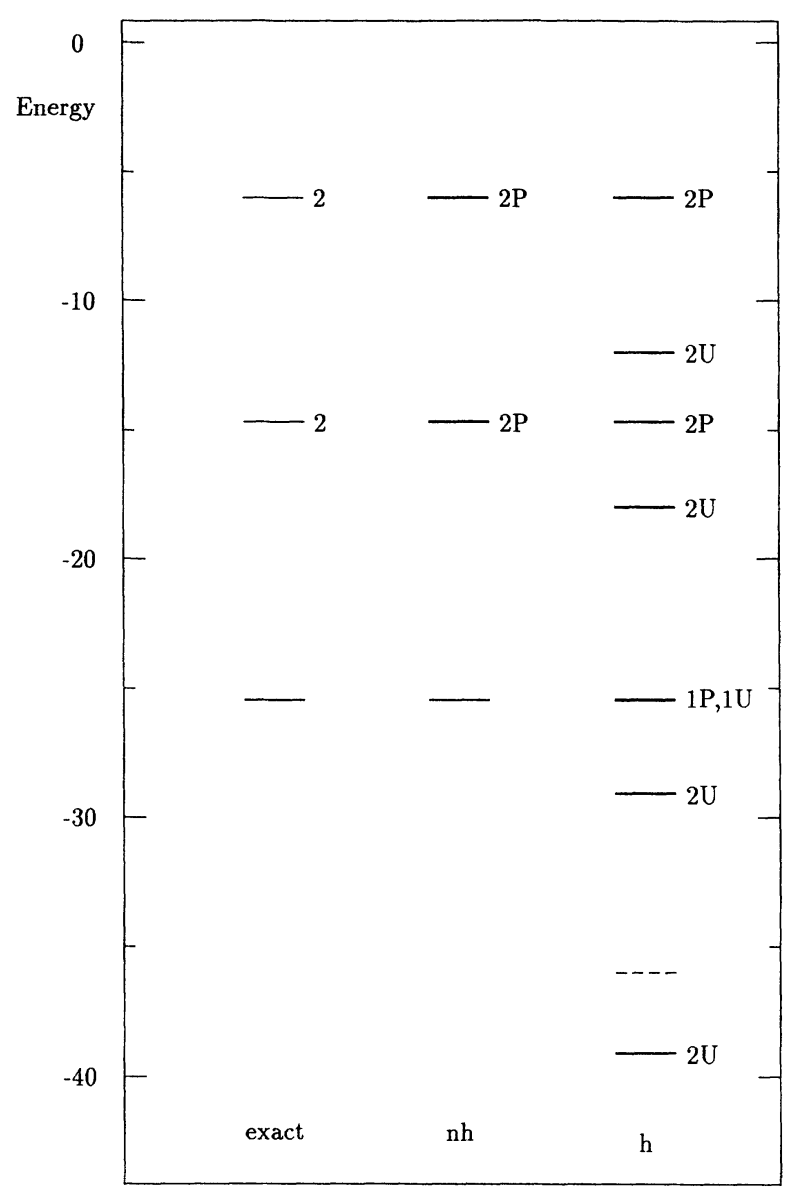

FIG. 2. The same as Fig. 1 except that the Hamiltonian parameters used are $\Delta=0, \chi_{2}=0$, and $\chi_{3}=1$.

The results presented in these three figures confirm that (at least for this model) our non-Hermitian baryon mapping provides a practical means of incorporating dynamical multiquark correlations in many-quark systems.

\section{SUMMARY AND CLOSING REMARKS}

We have presented in this paper a colorless mapping of three-quark clusters onto triplet fermions that is specifically tailored to physical constituent quark models in which the Hamiltonian may contain up to three-quark interactions and is a color scalar. Most notably, by concentrating on the mapping of colorless operators, we were able to achieve a unique prescription for a non-Hermitian mapping of two-quark interactions, the lack of which has been a limitation of earlier efforts.

We have also developed a three-color extension of the Lipkin model to assess the validity of our mapping. This model admits dynamical one-, two-, and three-body correlations, and can thus test the mapping for a variety of physically important scenarios. Furthermore, it can be solved exactly by purely algebraic means for small values of $\Omega$, the degeneracy of each Lipkin level. For $\Omega=2$, it can be mapped (using either our non-Hermitian or the simpler Hermitian form) onto a model of color- 


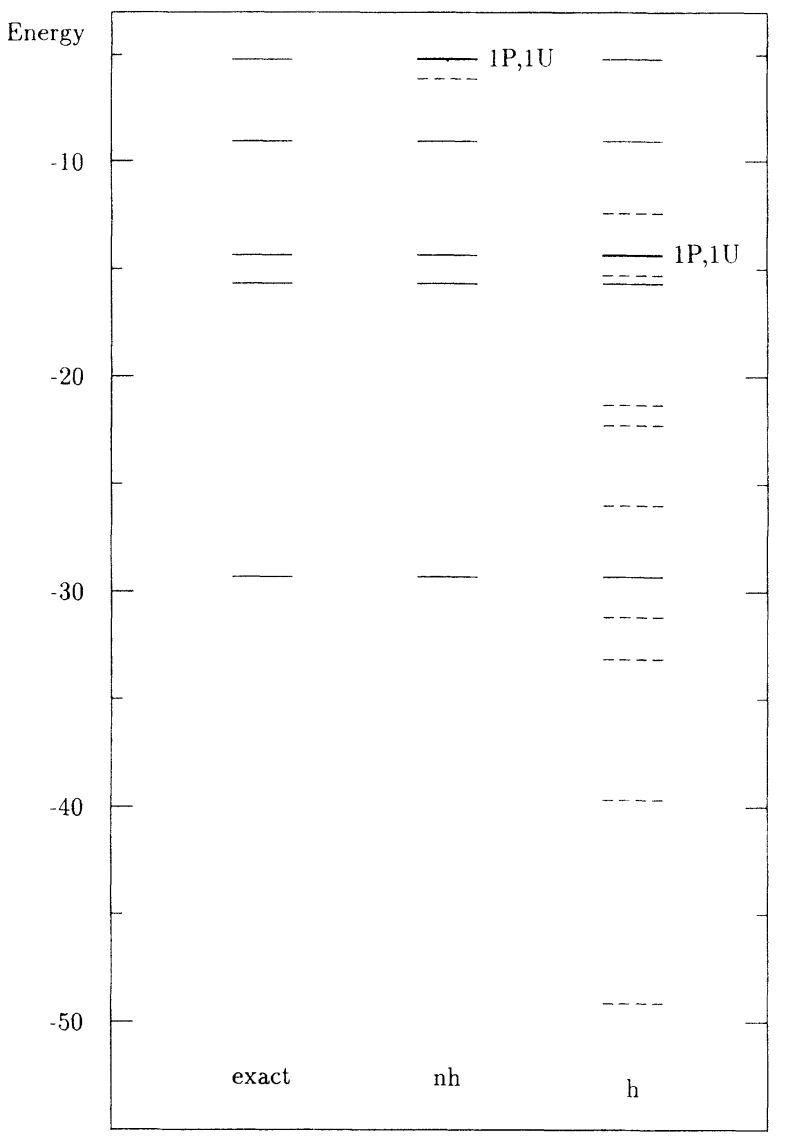

FIG. 3. The same as Fig. 1 except that the Hamiltonian parameters used are $\Delta=1, \chi_{2}=1$, and $\chi_{3}=1$ and the spectrum is limited to levels with $E<-5$.

less baryons and then diagonalized exactly. We find that our mapping exactly reproduces (within its physical subspace) the properties of all states of the original quark model. Furthermore, when the mapping is implemented in its non-Hermitian version, the unphysical states (those that are a pure artifact of the mapping and have no physical relevance) are pushed up in energy, and the lowest mapped eigenstates are physical.

For many-triplet systems (either based on the Lipkin model or a more realistic constituent quark model), exact diagonalization of the effective triplet Hamiltonian after the mapping is impossible. In such cases approximate many-body techniques, often based on variational meth- ods, are required. The fact that the unphysical states are pushed up in energy by the non-Hermitian mapping is crucial in this regard. Once an approximate diagonalization is carried out, the strict separation between physical and unphysical states is lost. It is essential, therefore, that the unphysical states be relatively high in energy, so that they do not mix appreciably into approximations to the low-lying states of interest.

To date, we have succeeded in solving the three-color Lipkin model by algebraic means for $\Omega=2$ and 3 only. Working in collaboration with Etienne Caurier, we have extended the range of exact solutions to $\Omega=6$, using large-basis shell-model calculations. However, we have not yet developed the necessary techniques to implement an approximate diagonalization of the mapped Hamiltonian for these larger values of $\Omega$. Clearly, such methods are essential if we wish to test the usefulness of our mapping for many-triplet systems, as would arise, e.g., in the description of finite nuclei. Work along these lines is currently underway.

The three-color Lipkin model that was developed to test our baryon mappings does not contain any spatial degrees of freedom and thus excludes spatial correlations. The only dynamical correlations in this model are those involving the $\sigma$ degree of freedom. It is important to further test our mapping in the context of solvable models that admit spatial three-quark clustering. This too is currently under investigation.

Finally, as was pointed out in the Introduction, fundamental questions remain regarding the use of nonrelativistic constituent quark models as a starting point for a description of finite nuclei. It is thus essential to try to establish a link between the nonrelativistic constituent quark picture and the underlying physics of QCD.

\section{ACKNOWLEDGMENTS}

This work was supported in part by the National Science Foundation under Grant Nos. PHY-9108011, PHY9303041, by NATO under Grant No. CRG.900466, by the Spanish DGICYT under Project No. PB92-0663, and by CONACYT, Mexico, under Project No. E-120.3475. One of the authors (A.F.) acknowledges the Guggenheim Foundation for its support during the development of this work. We would also like to express our appreciation to Etienne Caurier who carried out shell-model calculations for the three-color Lipkin model that verified the algebraic results reported here.
[1] M. Oka, K. Shimizu, and Y. Yazaki, Nucl. Phys. A464, 700 (1987); K. Brauer, A. Faessler, F. Fernandez, and K. Shimizu, ibid. A507, 599 (1990).

[2] Y. Yamauchi, A. Buchmann, A. Faessler, and A. Arima, Nucl. Phys. A526, 495 (1991).

[3] S. Pittel, J. Engel, J. Dukelsky, and P. Ring, Phys. Lett. B 247, 185 (1990).

[4] E. G. Nadjakov, J. Phys. G 16, 1473 (1990).

[5] J. Meyer, J. Math. Phys. 328, 2142 (1991).

[6] Abraham Klein and E. R. Marshalek, Rev. Mod. Phys. 63, 375 (1991).
[7] P. Ring and P. Schuck, The Nuclear Many-Body Problem (Springer, Berlin, 1980).

[8] H. R. Petry, H. Hofestädt, S. Merk, K. Bleuler, H. Bohr, and K. S. Narain, Phys. Lett. 159B, 363 (1985); H. Hofestädt, S. Merk, and H. R. Petry, Z. Phys. A 326, 391 (1987).

[9] Y. Suzuki and K. T. Hecht, Phys. Lett. B 232, 159 (1989).

[10] H. J. Lipkin, N. Meshkov, and A. Glick, Nucl. Phys. 62, 188 (1965).

[11] P. Park, Phys. Rev. C 35, 807 (1987). 